\title{
ON THE BEHAVIOR OF MAPPINGS WHICH DO NOT SATISFY HYERS-ULAM STABILITY
}

\author{
THEMISTOCLES M. RASSIAS AND PETER ŠEMRL
}

(Communicated by Palle E. T. Jorgensen)

\begin{abstract}
We find an example to show when the Hyers-Ulam stability does not occur for approximately linear mappings. We also investigate the behavior of such mappings.
\end{abstract}

Let $E_{1}, E_{2}$ be two real Banach spaces, and let $f: E_{1} \rightarrow E_{2}$ be a mapping that is "approximately linear." S. M. Ulam posed the following problem: "Give conditions in order for a linear mapping near an approximately linear mapping to exist" $[5,6]$. The solution to this problem has been given in the following way:

Theorem 1. Consider $E_{1}, E_{2}$ to be two Banach spaces, and let $f: E_{1} \rightarrow E_{2}$ be a mapping such that $f(t x)$ is continuous in $t$ for each fixed $x$. Assume that there exist $\theta \geq 0$ and $p \neq 1$ such that

$$
\|f(x+y)-f(x)-f(y)\| \leq \theta\left(\|x\|^{p}+\|y\|^{p}\right), \quad \text { for any } x, y \in E_{1} .
$$

Then there exists a unique linear mapping $T: E_{1} \rightarrow E_{2}$ such that, in the case $p<1$,

$$
\|f(x)-T(x)\| \leq \frac{2 \theta}{2-2^{p}}\|x\|^{p}, \quad \text { for any } x \in E_{1},
$$

while in the case $p>1$,

$$
\|f(x)-T(x)\| \leq \frac{2 \theta}{2^{p}-2}\|x\|^{p}, \quad \text { for any } x \in E_{1} .
$$

The solution to this problem was obtained by D. H. Hyers for $p=0$ [2]. This result was generalized by T. M. Rassias for $p \in[0,1)$ [3]. One can easily verify that the proof given in [3] also works when $p<0$. The problem for $p>1$ was solved by Z. Gajda using a similar approach [1]. This problem was further considered in [4]. In this article we shall give a very simple example to show that a stability theorem cannot be proved for $p=1$. We shall show that there exists a continuous real-valued function $f: \mathbb{R} \rightarrow \mathbb{R}$ satisfying

$$
|f(x+y)-f(x)-f(y)| \leq|x|+|y|,
$$

Received by the editors July 3, 1990.

1980 Mathematics Subject Classification (1985 Revision). Primary 47H15.

Key words and phrases. Approximately linear mapping.

The second author was partly supported by the Research Council of Slovenia. 
for all $x, y \in \mathbb{R}$, such that

$$
\lim _{x \rightarrow \infty} \frac{f(x)}{x}=\infty
$$

It follows that the set $\{|f(x)-T(x)| /|x| ; x \neq 0\}$ is unbounded for any linear mapping $T: \mathbb{R} \rightarrow \mathbb{R}$ defined by $T(x)=a x$ for a given $a \in \mathbb{R}$. In other words, an analogue of Theorem 1 for $p=1$ cannot be obtained. In [1], Gajda succeeded in constructing an example of a bounded continuous function $g: \mathbb{R} \rightarrow \mathbb{R}$ satisfying

$$
|g(x+y)-g(x)-g(y)| \leq|x|+|y|,
$$

for all $x, y \in \mathbb{R}$, with

$$
\lim _{x \rightarrow 0} \frac{g(x)}{x}=\infty
$$

The function $f(x)$ is far from being linear when $x$ tends to infinity, while the function $g(x)$ behaves badly near 0 . One can construct new examples by taking any linear combination of $f$ and $g$.

We shall also investigate the behavior of approximately linear mappings between Euclidean spaces.

Theorem 2. There exists a continuous function $f: \mathbb{R} \rightarrow \mathbb{R}$ satisfying

$$
|f(x+y)-f(x)-f(y)| \leq|x|+|y|,
$$

for any $x, y \in \mathbb{R}$, with

$$
\lim _{x \rightarrow \infty} \frac{f(x)}{x}=\infty
$$

Proof. The function $f$ defined by

$$
f(x)= \begin{cases}x \log _{2}(x+1) & \text { if } x \geq 0, \\ x \log _{2}|x-1| & \text { if } x<0\end{cases}
$$

satisfies the condition $\lim _{x \rightarrow \infty} \frac{f(x)}{x}=\infty$. One can easily verify that it is continuous, odd, and convex on the set of positive real numbers. Let $x, y$ be any two positive real numbers. Since $f$ is convex, it follows that

$$
|f(x+y)-f(x)-f(y)| \leq f(c)-2 f(c / 2),
$$

where $c=x+y$. From (2) and (3), inequality (1) follows. Since $f$ is an odd function, this inequality holds for $x, y<0$ as well. It remains to consider the case when $x>0$ and $y<0$. There is no loss of generality in assuming that $|x|>|y|$ holds. Because $f$ is odd and convex on the set $\mathbb{R}^{+}$, we have

$|f(x+y)-f(x)-f(y)|=-f(x+y)+f(x)+f(y)=f(x)-f(x+y)-f(-y)$.

But $x+y$ and $-y$ are positive real numbers. Thus, the previous argument completes the proof. Q.E.D.

In the following theorem we shall investigate the behavior of mappings that are approximately linear in the sense of inequality (1).

Theorem 3. Let $f: \mathbb{R}^{k} \rightarrow \mathbb{R}^{l}$ be a mapping such that $f(t x)$ is continuous in $t$ for each fixed $x$, where $k$ and $l$ are given positive integers. Assume that there exists $\theta \geq 0$ such that

$$
\|f(x+y)-f(x)-f(y)\| \leq \theta(\|x\|+\|y\|), \quad \text { for any } x, y \in \mathbb{R}^{k} .
$$


Then for every positive real number $p$, there exists a real number $M_{p}$ such that

$$
\|f(x)\| \leq M_{p}\|x\|^{1+p}, \quad \text { if }\|x\| \geq 1,
$$

and

$$
\|f(x)\| \leq M_{p}\|x\|^{1-p}, \quad \text { if }\|x\| \leq 1 .
$$

Proof. Applying (4) and induction on $n$, one can prove that

$$
\begin{aligned}
& \left\|f\left(x_{1}+x_{2}+\cdots+x_{n}\right)-f\left(x_{1}\right)-f\left(x_{2}\right)-\cdots-f\left(x_{n}\right)\right\| \\
& \leq \theta(n-1)\left(\left\|x_{1}\right\|+\left\|x_{2}\right\|+\cdots+\left\|x_{n}\right\|\right) .
\end{aligned}
$$

Let $\left\{e_{1}, e_{2}, \ldots, e_{k}\right\}$ be the standard basis in $\mathbb{R}^{k}$. An arbitrary vector $x \in \mathbb{R}^{k}$ with $\|x\| \leq 1$ can be expressed in the form

$$
x=\lambda_{1} e_{1}+\lambda_{2} e_{2}+\cdots+\lambda_{k} e_{k},
$$

where $\left|\lambda_{i}\right| \leq 1$ for $i=1,2, \ldots, k$. It follows from (7) that

$$
\begin{gathered}
\left\|f\left(\lambda_{1} e_{1}+\lambda_{2} e_{2}+\cdots+\lambda_{k} e_{k}\right)-f\left(\lambda_{1} e_{1}\right)-f\left(\lambda_{2} e_{2}\right)-\cdots-f\left(\lambda_{k} e_{k}\right)\right\| \\
\leq \theta(k-1)\left(\left\|\lambda_{1} e_{1}\right\|+\left\|\lambda_{2} e_{2}\right\|+\cdots+\left\|\lambda_{k} e_{k}\right\|\right) \leq \theta(k-1) k .
\end{gathered}
$$

Then

$$
\begin{aligned}
\left\|f\left(\lambda_{1} e_{1}+\lambda_{2} e_{2}+\cdots+\lambda_{k} e_{k}\right)\right\| \leq & \theta(k-1) k+\left\|f\left(\lambda_{1} e_{1}\right)\right\| \\
& +\left\|f\left(\lambda_{2} e_{2}\right)\right\|+\cdots+\left\|f\left(\lambda_{k} e_{k}\right)\right\| \\
\leq & \theta(k-1) k+M_{1}+M_{2}+\cdots+M_{k},
\end{aligned}
$$

where $M_{i}=\max _{|\lambda| \leq 1}\left\|f\left(\lambda e_{i}\right)\right\|$. Hence, $f$ is bounded on the unit ball in $\mathbb{R}^{k}$. Thus, there exists a real number $c$ such that

$$
\|f(x)\| \leq c\|x\|
$$

for all $x$ satisfying $\frac{1}{2} \leq\|x\| \leq 1$.

Claim that

$$
\left\|2^{-n} f\left(2^{n} x\right)-f(x)\right\| \leq n \theta\|x\|
$$

for any positive integer $n$. The verification of (9) follows by induction on $n$. According to the hypothesis, the case $n=1$ is clear. Assume now that (9) holds and we want to prove it for the case $(n+1)$. Using the triangle inequality and (9), we get

$$
\begin{aligned}
\left\|2^{-n-1} f\left(2^{n+1} x\right)-f(x)\right\| & \leq\left\|2^{-n-1} f\left(2^{n} 2 x\right)-2^{-1} f(2 x)\right\|+\left\|2^{-1} f(2 x)-f(x)\right\| \\
& \leq(n+1) \theta\|x\| .
\end{aligned}
$$

For any $x$ with norm greater than one, we can find an integer $n$ such that the vector $y=2^{-n} x$ satisfies $\frac{1}{2} \leq\|y\| \leq 1$. Moreover, we have $n \leq \log _{2}\|x\|+1$. It follows from (9) that

$$
\left\|2^{-n} f(x)-f(y)\right\| \leq n \theta\|y\| .
$$

Therefore

$$
\|f(x)\| \leq 2^{n}(\|f(y)\|+n \theta\|y\|) \leq 2^{n}\|y\|(c+n \theta) \leq\|x\|\left(c+\theta\left(\log _{2}\|x\|+1\right)\right),
$$

which proves $(5)$. 
A similar argument as in the proof of (9) yields

$$
\left\|2^{n} f\left(2^{-n} x\right)-f(x)\right\| \leq n \theta\|x\|
$$

for any positive integer $n$. For any $x$ in the unit ball $\|x\| \leq 1$, there exists an integer $n$ such that the vector $y=2^{n} x$ satisfies $\frac{1}{2} \leq\|y\| \leq 1$. It follows that $n \leq-\log _{2}\|x\|$. As in the previous case, we obtain

$$
\left\|2^{n} f(x)-f(y)\right\| \leq n \theta\|y\| .
$$

Thus,

$$
\|f(x)\| \leq 2^{-n}(\|f(y)\|+n \theta\|y\|) \leq\|x\|\left(c-\theta \log _{2}\|x\|\right) .
$$

Hence, (6) holds. Q.E.D.

Remarks. (I) Theorem 3 cannot be generalized for mappings defined on an arbitrary Banach space. In fact, every unbounded linear operator $f: E_{1} \rightarrow E_{2}$ satisfies (4). However, conditions (5) and (6) are not fulfilled.

(II) The same proof works for mappings defined on an arbitrary Euclidean space with values in an arbitrary Banach space.

We conclude with an extension of Theorem 1.

Theorem 4. Consider $E_{1}, E_{2}$ to be two Banach spaces, and let $f: E_{1} \rightarrow E_{2}$ be a mapping such that $f(t x)$ is continuous in $t$ for each fixed $x$. Let $k$ be a positive integer, $k \geq 2$. Assume that there exist $\theta \geq 0$ and $p \neq 1$ such that

$$
\left\|f\left(\sum_{i=1}^{k} x_{i}\right)-\sum_{i=1}^{k} f\left(x_{i}\right)\right\| \leq \theta\left(\sum_{i=1}^{k}\left\|x_{i}\right\|^{p}\right), \quad \text { for any } x_{1}, x_{2}, \ldots, x_{k} \in E_{1} \text {. }
$$

Then there exists a unique linear mapping $T: E_{1} \rightarrow E_{2}$ such that, in the case $p<1$,

$$
\|f(x)-T(x)\| \leq \frac{k \theta}{k-k^{p}}\|x\|^{p}, \quad \text { for any } x \in E_{1},
$$

while in the case $p>1$,

$$
\|f(x)-T(x)\| \leq \frac{k \theta}{k^{p}-k}\|x\|^{p}, \quad \text { for any } x \in E_{1} .
$$

Proof. One applies the same argument as in [3]. Q.E.D.

\section{ACKNOWLEDGMENT}

We wish to express our gratitude to Professor Časlav Stanojević for inviting us to the Fourth International Workshop in Analysis and Its Applications, Dubrovnik-Kupari, Yugoslavia, June, 1990, during which time this work was completed.

\section{REFERENCES}

1. Z. Gajda, On isometric mappings, Internat. J. Math. Math. Sci. (to appear).

2. D. H. Hyers, On the stability of the linear functional equation, Proc. Nat. Acad. Sci. U.S.A. 27 (1941), 222-224.

3. T. M. Rassias, On the stability of the linear mapping in Banach spaces, Proc. Amer. Math. Soc. 72 (1978), 297-300.

4. __ On a modified Hyers-Ulam sequence, J. Math. Anal. Appl. (to appear). 
5. S. M. Ulam, Problems in modern mathematics, Chapter VI, Wiley, New York, 1960.

6. __ Sets, numbers, and universes, Selected Works, Part III, MIT Press, Cambridge, MA and London, 1974.

Department of Mathematics, University of La Verne, P. O. BoX 51105, Kifissia, Athens, GREECE 14510

Department of Mathematics, E. K. University of Ljubluana, Jadranska 19, 61000 LJubluana, Yugoslavia 